\title{
Body-object interaction ratings for 1,618 monosyllabic nouns
}

\author{
Sherri M. Tillotson and Paul D. Siakaluk \\ University of Northern British Columbia, Prince George, British Columbia, Canada \\ AND \\ Penny M. Pexman \\ University of Calgary, Alberta, Canada
}

\begin{abstract}
Body-object interaction (BOI) assesses the ease with which a human body can physically interact with a word's referent. Recent research has shown that BOI influences visual word recognition processes in such a way that responses to high-BOI words (e.g., couch) are faster and less error prone than responses to low-BOI words (e.g., cliff ). Importantly, the high-BOI words and the low-BOI words that were used in those studies were matched on imageability. In the present study, we collected BOI ratings for a large set of words. BOI ratings, on a 1-7 scale, were obtained for 1,618 monosyllabic nouns. These ratings allowed us to test the generalizability of BOI effects to a large set of items, and they should be useful to researchers who are interested in manipulating or controlling for the effects of BOI. The body-object interaction ratings for this study may be downloaded from the Psychonomic Society's Archive of Norms, Stimuli, and Data, www.psychonomic.org/archive.
\end{abstract}

The importance of sensorimotor interactions with the environment to the development and structure of cognitive processes is now being taken seriously by an increasing number of cognitive scientists. An embodied cognition approach to the study of cognitive processing has been adopted by researchers examining such varied abilities as cognitive and motor development, mental imagery, memory, reasoning and problem solving, linguistic processing, metaphor processing, and concept formation (Clark, 1997; Gibbs, 2006; Glenberg, 1997; Lakoff \& Johnson, 1999; Pecher \& Zwaan, 2005; Wilson, 2002).

In the research area of visual word recognition, it could be proposed that imageability effects (i.e., words with referents that can be easily sensed, such as peach, are recognized more rapidly and/or more accurately than words with referents that cannot be easily sensed, such as fraud) (e.g., Cortese, Simpson, \& Woolsey, 1997; Strain, Patterson, \& Seidenberg, 1995) are best understood from an embodied cognitive perspective. As a result of repeated sensory experience with certain objects or events, words that refer to these objects or events develop richer linguistic representations than do words that refer to objects or events with which humans have less sensory experience. According to an embodied cognitive perspective, an important component of the conceptual understanding of easily imageable words is the set of sensory representations that may be activated when linguistic processing occurs, resulting in faster and more accurate responses to these words in visual word recognition tasks.
Barsalou and colleagues recently developed an embodied framework that they described as perceptual symbol systems theory (Barsalou, 1999, 2003a, 2003b; Barsalou, Simmons, Barbey, \& Wilson, 2003). According to this theory, many different modalities are involved in the acquisition and processing of conceptual knowledge. In addition to systems that have been well studied in cognitive psychology, such as cognitive systems (e.g., attention and language processing) and sensory systems (e.g., vision and olfaction), the theory suggests that less studied systems, such as motor, kinesthetic, and proprioceptive systems (e.g., grasping, manual manipulation, and internal feedback from muscles and joints) and emotional systems (e.g., fear and pleasure), are fundamental to concept formation and processing. Although knowledge acquired through these individual systems is at first processed in modalityspecific memory systems, it is eventually fed into hierarchical convergence areas where integration of the separate forms of information takes place (see also Damasio, 1989; Edelman \& Tononi, 2000; Kandel, 2006; LeDoux, 2001). Accessing conceptual knowledge involves partial activation or simulation of the varied systems that were involved in the original encoding of the object or event. According to this perspective, sensory and motor knowledge gained via bodily experience with the environment is an important part of what is learned about concepts, and it is accessible to the linguistic system.

Recently, we examined the influence on visual word recognition of a variable that we call body-object inter-

P. D. Siakaluk, siakaluk@unbc.ca 
action (BOI). BOI assesses perceptions of the ease with which a human body can physically interact with a word's referent. We reported that responses to words that were rated high in BOI were faster and more accurate than responses to words that were rated low in BOI in lexical decision and phonological lexical decision tasks (Siakaluk, Pexman, Aguilera, Owen, \& Sears, 2008) and in several variants of the semantic categorization task (Siakaluk, Pexman, Sears, et al., 2008). Importantly, the words that were rated high and low in BOI were matched on imageability (and on the related dimension of concreteness, in addition to other lexical and semantic variables). Thus, the facilitatory processing of high-BOI words was clearly independent of the effects of imageability.

Some researchers (e.g., Gibbs, 2006) question whether sensory and motor effects can be sufficiently disentangled. If one assumes, however, that BOI effects reflect motor experience with words' referents, whereas imageability effects reflect sensory experience with words' referents, then our reports of facilitatory BOI effects that were independent of facilitatory imageability effects lend initial support to the notion that these dimensions can be disentangled, at least in the research domain of visual word recognition.

We thought that it was important to collect BOI ratings for a large set of words in order to (1) test whether BOI effects generalize to a larger set of items than has been used in the previous research, (2) investigate whether imageability and BOI effects explain unique variance in visual word recognition, and (3) provide a set of BOI ratings to allow researchers to manipulate or control for the effect of BOI.

\section{METHOD}

\section{Participants}

Ninety undergraduate students participated in the study for bonus course credit. Half of the participants were undergraduate students at the University of Northern British Columbia, and half were undergraduate students at the University of Calgary. All participants were native English speakers, and all reported normal or correctedto-normal vision.

\section{The Word Corpus}

For the study, 1,620 monosyllabic words were selected from the Cortese and Fugett (2004) imageability corpus. All of the words had noun definitions that were listed first in the ITP Nelson Canadian Dictionary (1997). Three word lists of 540 nouns were subsequently constructed, and each list was matched on printed frequency, word length, and imageability (all $p \mathrm{~s}>.20){ }^{1}$

\section{Apparatus and Procedure}

Thirty participants - half at the University of Northern British Columbia and half at the University of Calgary - provided BOI ratings for each word list. At the University of Northern British Columbia, the words were presented in the center of a 17-in. monitor that was driven by a Pentium-class microcomputer running DirectRT software (www.empirisoft.com/DirectRT.aspx). At the University of Calgary, the words were presented in the center of a 17-in. monitor that was driven by a Macintosh G3 computer using PsyScope (Cohen, MacWhinney, Flatt, \& Provost, 1993).

Each trial was initiated by a fixation marker that appeared at the center of the computer display. The fixation marker was presented for 1,000 msec and was then replaced by a word. The participants' task was to read the word and then rate how easily a human body can physically interact with the word's referent (see the Appendix). Participants entered their ratings by using the numbered keypad on the right side of the keyboard. The stimulus order was randomized separately for each participant, and the intertrial interval was 2,000 msec. Participants were given an opportunity to take a break after every 180 trials.

\section{Data Analysis}

The data were analyzed using standard multiple regression.

\section{RESULTS AND DISCUSSION}

Data were screened in the same manner as in Cortese and Fugett (2004): Ratings were removed from the data set for trials on which the rating was made in less than 300 msec. Participants were not instructed to make their ratings as rapidly as possible, so no maximum time limit was applied. A total of 363 observations $(0.75 \%$ of the data) were removed as a result of this procedure.

Analyses of the ratings data showed that the mean BOI rating was $3.27(S D=1.42)$. The mean imageability rating for this set of words in the Cortese and Fugett (2004) norms was $4.63(S D=1.45)$. As we expected, there was a strong positive correlation between the ratings for BOI and imageability $[r(1,617)=.79, p<.001]$. We next examined whether these two variables were unique predictors of variance in visual word recognition. Importantly, we expected facilitatory effects of BOI for only moderately to highly imageable words. This is because human bodies can interact only with concrete concepts.

We examined this question by conducting multiple regression analyses on lexical decision response latencies and accuracy rates that were obtained from the English Lexicon Project database (Balota et al., 2007) for all items that were rated higher than the midpoint of the imageability scale that was used by Cortese and Fugett (2004) (i.e.,

Table 1

Zero-Order Correlations Between Predictor Variables and Measures of Lexical Decision Task Performance

\begin{tabular}{lcccccccc}
\hline \multicolumn{1}{c}{ Measure } & 1 & 2 & 3 & 4 & 5 & 6 & 7 & 8 \\
\hline 1. HAL log frequency & - & & & & & & & \\
2. Printed length & $-.12^{*}$ & - & & & & & & \\
3. Orthographic neighborhood size & $.16^{*}$ & $-.71^{*}$ & - & & & & \\
4. Phonological neighborhood size & $.18^{*}$ & $-.47^{*}$ & $.64^{*}$ & - & & & \\
5. Imageability & $.21^{*}$ & $-.14^{*}$ & $.09^{*}$ & $.11^{*}$ & - & & \\
6. Body-object interaction & $.10^{*}$ & $-.14^{*}$ & $.15^{*}$ & $.15^{*}$ & $.67^{*}$ & - & & \\
7. LDT response latency & $-.62^{*}$ & $.14^{*}$ & $-.16^{*}$ & $-.15^{*}$ & $-.31^{*}$ & $-.24^{*}$ & - & \\
8. LDT error & $.49^{*}$ & -.00 & .05 & -.00 & $.33^{*}$ & $.24^{*}$ & $-.61^{*}$ & - \\
\hline Note-HAL, hyperspace analogue to language; LDT, lexical decision task ${ }^{*} p<.01$ & & &
\end{tabular}

Note-HAL, hyperspace analogue to language; LDT, lexical decision task. ${ }^{*} p<.01$ 
Table 2

Results of Standard Multiple Regression Analyses for Lexical Decision Task Performance for All Items With Imageability Ratings Greater Than 4.0 Out of 7.0

\begin{tabular}{|c|c|c|c|c|}
\hline Variable & $B$ & $S E(B)$ & $\beta$ & $s r$ \\
\hline \multicolumn{5}{|c|}{ Response Latency $\left(R^{2}=.42^{* * *}\right)$} \\
\hline HAL log frequency & -22.27 & 0.97 & $-.58^{* * *}$ & -.56 \\
\hline Printed length & 1.47 & 2.62 & .02 & .01 \\
\hline Orthographic neighborhood size & -0.31 & 0.38 & -.03 & -.02 \\
\hline Phonological neighborhood size & 0.07 & 0.15 & .01 & .01 \\
\hline Imageability & -9.12 & 2.65 & $-.12^{* *}$ & -.08 \\
\hline Body-object interaction & -4.88 & 1.66 & $-.10^{* *}$ & -.07 \\
\hline \multicolumn{5}{|c|}{$\operatorname{Error}\left(R^{2}=.31^{* * *}\right)$} \\
\hline HAL log frequency & 0.02 & 0.00 & $.46^{* * *}$ & .44 \\
\hline Printed length & 0.01 & 0.00 & $.09^{*}$ & .06 \\
\hline Orthographic neighborhood size & 0.00 & 0.00 & $.10^{*}$ & .06 \\
\hline Phonological neighborhood size & -0.00 & 0.00 & $-.14^{* * *}$ & -.10 \\
\hline Imageability & 0.02 & 0.00 & $.19^{* * *}$ & .14 \\
\hline Body-object interaction & 0.01 & 0.00 & $.09^{*}$ & .06 \\
\hline
\end{tabular}

Note-HAL, hyperspace analogue to language. $B$ is the unstandardized coefficient; $s r$, the semipartial correlation. ${ }^{*} p<.05 .{ }^{* *} p<$ $.01{ }^{* * *} p<.001$.

all items that were rated higher than 4.0 out of 7.0). There were 987 such items from our set that were included in the analyses. The following variables were included as predictors: log-transformed hyperspace analogue to language frequency, printed length, orthographic neighborhood size, phonological neighborhood size, imageability ratings, and BOI ratings (the first four predictors were obtained from the English Lexicon Project database). ${ }^{2}$ Zero-order correlations between predictor variables and measures of performance are shown in Table 1. The multiple regression results are shown in Table 2. Of most importance for the present study is the fact that for moderately to highly imageable words, BOI accounted for a significant amount of unique variability for both response latency and accuracy rate, despite its strong positive correlation with imageability (for the response latency results, the magnitude of the BOI effect was similar to that of imageability). ${ }^{3}$

In summary, these results are important because they suggest that facilitatory BOI effects extend to a much larger set of items than those that have been used in previous studies. Furthermore, the present results confirm that BOI and imageability make independent contributions to visual word recognition for words that are rated moderately to highly imageable. One implication of this result is the possibility that previously reported facilitatory effects of imageability may be partially attributable to the influence of BOI. Further research is needed to better disentangle the effects of these two variables, and to extend the investigation to include multisyllabic words. The BOI ratings for the complete set of words that were collected in the present study should prove useful for researchers who are interested in examining or controlling for the effects of BOI in visual word recognition and in other areas of cognitive research.

\section{AUTHOR NOTE}

This research was supported by Natural Sciences and Engineering Research Council of Canada (NSERC) Discovery Grants to P.D.S. and
P.M.P., and by an NSERC Undergraduate Student Research Award to S.M.T. Correspondence concerning this article should be addressed to P. D. Siakaluk, Department of Psychology, University of Northern British Columbia, Prince George, BC, V2N 4Z9 Canada (e-mail: siakaluk@, unbc.ca).

\section{REFERENCES}

Balota, D. A., Yap, M. J., Cortese, M. J., Hutchison, K. A., KessLER, B., LofTIS, B., ET AL. (2007). The English Lexicon Project. Behavior Research Methods, 39, 445-459.

Barsalou, L. W. (1999). Perceptual symbol systems. Behavioral \& Brain Sciences, 22, 577-660.

BARSALOU, L. W. (2003a). Abstraction in perceptual symbol systems. Philosophical Transactions of the Royal Society B, 358, 1177-1187.

BARSALOU, L. W. (2003b). Situated simulation in the human conceptual system. Language \& Cognitive Processes, 18, 513-562.

Barsalou, L. W., Simmons, W. K., Barbey, A. K., \& Wilson, C. D. (2003). Grounding conceptual knowledge in modality-specific systems. Trends in Cognitive Sciences, 7, 84-91.

Clark, A. (1997). Being there: Putting brain, body, and world together again. Cambridge, MA: MIT Press.

Cohen, J., MacWhinney, B., Flatt, M., \& Provost, J. (1993). PsyScope: An interactive graphic system for designing and controlling experiments in the psychology laboratory using Macintosh computers. Behavior Research Methods, Instruments, \& Computers, 25, 257-271.

Cortese, M. J., \& FugetT, A. (2004). Imageability ratings for 3,000 monosyllabic words. Behavior Research Methods, Instruments, \& Computers, 36, 384-387.

Cortese, M. J., Simpson, G. B., \& Woolsey, S. (1997). Effects of association and imageability on phonological mapping. Psychonomic Bulletin \& Review, 4, 226-231.

DAMAsio, A. R. (1989). Time-locked multiregional retroactivation: A systems-level proposal for the neural substrates of recall and recognition. Cognition, 33, 25-62.

Edelman, G. M., \& Tononi, G. (2000). A universe of consciousness: How matter becomes imagination. New York: Basic Books.

GibBs, R. W. (2006). Embodiment and cognitive science. New York: Cambridge University Press.

Glenberg, A. M. (1997). What memory is for. Behavioral \& Brain Sciences, 20, 1-55.

ITP Nelson Canadian dictionary of the English language: An encyclopedic reference (1997). Toronto: ITP Nelson.

KANDEL, E. R. (2006). In search of memory: The emergence of a new science of mind. New York: W. W. Norton.

Lakoff, G., \& Johnson, M. (1999). Philosophy in the flesh: The embodied mind and its challenge to western thought. New York: Basic Books.

LeDoux, J. E. (2001). Synaptic self: How our brains become who we are. New York: Viking.

Pecher, D., \& ZwaAn, R. A. (Eds.) (2005). Grounding cognition: The role of perception and action in memory, language, and thinking. Cambridge: Cambridge University Press.

Siakaluk, P. D., Pexman, P. M., Aguilera, L., Owen, W. J., \& Sears, C. R. (2008). Evidence for the activation of sensorimotor information during visual word recognition: The body-object interaction effect. Cognition, 106, 433-443.

Siakaluk, P. D., Pexman, P. M., Sears, C. R., Wilson, K., Locheed, K., \& OWEN, W. J. (2008). The benefits of sensorimotor knowledge: Body-object interaction facilitates semantic processing. Cognitive Science, 32, 591-605.

Strain, E., Patterson, K., \& Seidenberg, M. S. (1995). Semantic effects in single-word naming. Journal of Experimental Psychology: Learning, Memory, \& Cognition, 21, 1140-1154.

WiLson, M. (2002). Six views of embodied cognition. Psychonomic Bulletin \& Review, 9, 625-636.

\section{NOTES}

1. One word from the first word list was used in the instructions used in the ratings task as the example of a high-BOI word. Another word from the third word list was entered into the stimulus file incorrectly. 
Thus, the responses to these two words were not included in the analyses, and the first and third word lists contributed 539 words to the analyses.

2. We chose to include these predictors because the values on these dimensions were available for all of the items in our list (all are available from the English Lexicon Project database), because each has been shown to influence word recognition performance, and because they capture the variance associated with the words' lexical and sublexical characteristics that we wanted to separate from BOI and imageability effects.

3 . As shown in Table 1, the correlation between rated BOI and imageability for the subset of words that were used in the multiple regression analysis was $r=.67$, which was lower than the correlation we observed for the entire set of words $(r=.79)$. Despite the strong relationship between these two predictors, collinearity diagnostics for the multiple regression analysis indicated that multicollinearity was not an issue for the analysis.

\section{ARCHIVED MATERIALS}

The following materials may be accessed through the Psychonomic Society's Norms, Stimuli, and Data archive, www.psychonomic.org/ archive.

To access these files, search the archive for this article using the journal (Behavior Research Methods), the corresponding author's name (Siakaluk), and the publication year (2008).

FILE: Siakaluk-BRM-2008.zip

DESCRIPTION: The compressed archive file contains two files:

Siakaluk(2008).txt, containing body-object interaction ratings.

Siakaluk(2008).xls, containing the above information in Excel spreadsheet format.

AUTHOR’S E-MAIL ADDRESS: siakaluk@unbc.ca.

\section{Written Instructions Used for the Body-Object Interaction Rating Task}

Words differ in the extent to which they refer to objects or things that a human body can physically interact with. Some words refer to objects or things that a human body can easily physically interact with, whereas other words refer to objects or things that a human body cannot easily physically interact with. The purpose of this experiment is to rate 540 words as to the ease with which a human body can physically interact with what they represent. For example, the word "chair" refers to an object or thing that a human body can easily physically interact with (e.g., a human body can sit on a chair, or stand on a chair, or move a chair from one part of a room to another), whereas the word "ceiling" refers to an object or thing that a human body cannot easily physically interact with (e.g., a human body could jump up and touch a ceiling). Any word (e.g., "chair") that in your estimation refers to an object or thing that a human body can easily physically interact with should be given a high body-object interaction rating (at the upper end of the numerical scale). Any word (e.g., "ceiling") that in your estimation refers to an object or thing that a human body cannot easily physically interact with should be given a low body-object interaction rating (at the lower end of the scale). It is important that you base these ratings on how easily a human body can physically interact with what a word represents, and not on how easily it can be experienced by human senses (e.g., vision, taste, etc.). Also, because words tend to make you think of other words as associates, it is important that your ratings not be based on this and that you judge only the ease with which a human body can physically interact with what a word represents. Remember, all the words are nouns (i.e., objects or things) and you should base your ratings on this fact.

Your body-object interaction ratings will be made on a 1 to 7 scale. A value of 1 will indicate a low body-object interaction rating, and a value of 7 will indicate a high body-object interaction rating. Values of 2 to 6 will indicate intermediate ratings. Please feel free to use the whole range of values provided when making your ratings. For each word, press the appropriate key on the keypad on the right of the keyboard when making your rating. When making your ratings, try to be as accurate as possible, but do not spend too much time on any one word.

$\begin{array}{lllrrrr}1 & 2 & 3 & 4 & 5 & 6 & 7 \\ \text { Low } & & & \text { Medium } & & & \text { High }\end{array}$

If you have any questions before the experiment begins, please ask the experimenter.

(Manuscript received November 2, 2007;

revision accepted for publication April 11, 2008.) 